\title{
TUBULAR SETS AND MULTIVARIATE POLYA ALGORITHM
}

\section{ROBERT HUOTARI}

(Received 11 January 1991; revised 10 October 1991)

Communicated by E. N. Dancer

\begin{abstract}
Some new results concerning tubular sets are presented, with applications to the convergence of the Polya algorithm in the contexts of simultaneous approximation and approximation of multivariate functions by univariate functions. (The Polya algorithm constructs a best uniform approximation from the limit, as $p \rightarrow \infty$, of best $L_{p}$ approximations.)
\end{abstract}

1991 Mathematics subject classification (Amer. Math. Soc.): 41 A 30, 41 A 63.

Keywords and phrases: Polya algorithm, multivariate approximation, tubular set.

In 1913, George Polya proposed an algorithm to calculate best uniform approximations to continuous functions by polynomials [8]. This algorithm utilizes the continuum of $L_{p}$ spaces, and is known as the Polya algorithm. Current usage of the Polya algorithm is primarily theoretical. In [2] it was used to show that best uniform approximations exist, in [6] its limit was shown to be a Lipschitz uniform selection operator, and in [3] and [8] its convergence was studied in new contexts. The theme underlying these studies is the evolution of $L_{p}$ to $L_{\infty}$ as $p \rightarrow \infty$. In [7], a sufficient condition for the convergence of the discrete Polya algorithm was presented. The present note continues the discussion of this condition and presents applications to the theory of multivariate approximation. In particular, it generalizes the simultaneous-Polya-algorithm-convergence result of [12], and it establishes the convergence of the Polya algorithm in a general context which includes the approximation of multivariate by univariate functions [1].

(C) 1993 Australian Mathematical Society $0263-6115 / 93 \$ A 2.00+0.00$ 


$$
\begin{aligned}
& \text { If } \mathbf{w}=\left(w_{1}, \ldots, w_{n}\right) \in \mathbb{R}^{n} \text { let } \\
& \qquad\|\mathbf{w}\|_{p}=\left(\sum_{i=1}^{n}\left|w_{i}\right|^{p}\right)^{1 / p}, \quad 1 \leq p<\infty \quad \text { and } \quad\|\mathbf{w}\|_{\infty}=\max _{1 \leq i \leq n}\left|x_{i}\right| .
\end{aligned}
$$

If $\mathbf{x} \in \mathbb{R}^{n}, K \subset \mathbb{R}^{n}$ and $1 \leq p \leq \infty$, we say that $\mathbf{x}^{*} \in K$ is a best $l_{p}$ approximation to $x$ from $K$ if

$$
\left\|\mathbf{x}-\mathbf{x}^{*}\right\|_{p}=\inf _{y \in K}\|\mathbf{x}-\mathbf{y}\|_{p}
$$

If $K$ is closed and convex, then for $1<p<\infty$, $\mathbf{x}$ has a unique best $l_{p}$ approximation, $\mathbf{x}^{p}$ from $K$ [13]. If $\lim _{p \rightarrow \infty} \mathbf{x}^{p}$ exists, the Polya algorithm converges. In this case we say that $K$ has the Polya property. (If $\lim \mathbf{x}^{p}$ exists, then the limit is a best $l_{\infty}$ approximation to $\mathbf{x}$ from $K$, so the algorithm is a method for constructing a best $l_{\infty}$ approximation.)

Our discussion of the Polya property will be facilitated by the following notations. If $A \subset \mathbb{R}^{n}$ let aff $(A), \operatorname{co}(A), \operatorname{ri}(A)$ and $\langle A\rangle$ denote, respectively, the affine hull, the convex hull and the relative interior of $A$, and the subspace of $\mathbb{R}^{n}$ generated by $A$. If $\mathbf{x} \in \mathbb{R}^{n}$ and $\alpha \in \mathbb{R}$, let $B(\mathbf{x}, \alpha)$ be the ball $\left\{\mathbf{y}\|\mathbf{y}-\mathbf{x}\|_{\infty}<\alpha\right\}$. If $\mathbf{a}, \mathbf{v} \in \mathbb{R}^{n}$ and $\delta \in \mathbb{R}$, let $C(A, \mathbf{v})=\{\mathbf{x}=\mathbf{a}+t \mathbf{v} \mathbf{a} \in A, t \in \mathbb{R}\}$, let $H_{\mathbf{a v}}$ be the hyperplane which contains a and is orthogonal to $\mathbf{v}$, and let $P_{\mathrm{av}}$ be the projection of $\mathbb{R}^{n}$ onto $H_{\mathrm{av}}$ along $\mathbf{V}$.

Suppose $K$ is closed and convex. If $\mathbf{a} \in K$ and $\mathbf{v} \in \mathbb{R}^{n}$, we say that $K$ is $\mathbf{v}$-tubular at $\mathbf{a}$, if for any $\varepsilon>0$ there exists $\delta=\delta(K, \mathbf{V}, \mathbf{a}, \varepsilon)>0$ such that, if $\mathbf{y} \in K$ and $\|\mathbf{a}+t \mathbf{v}-\mathbf{y}\|_{\infty}<\delta$ for some $t \in \mathbb{R}$, then there exists $s \in \mathbb{R}$ such that $\mathbf{z}:=\mathbf{y}+s \mathbf{v} \in K$ and $\|\mathbf{z}-\mathbf{z}\|_{\infty}<\varepsilon$. (This definition is equivalent to that of $\mathbf{v}$-cylindrical in [7].) In this case, we call $\mathbf{z}$ an $\varepsilon$-admissible replacement for $\mathbf{y}$. We say that $K$ is basically (respectively, totally) tubular if it is $\mathbf{v}$-tubular for every $\mathbf{v}$ in the standard basis for $\mathbb{R}^{n}$ (respectively, for every $\mathbf{v}$ in $\mathbb{R}^{n}$ ). All polygonal convex sets and all smooth rotund convex bodies are totally tubular [7].

A geometric view of the above definition may be useful. Suppose $\mathbf{v} \neq \mathbf{0}$. Then $K$ is v-tubular at $\mathbf{a}$ if and only if there is a function $Q: P_{\mathbf{a v}}(K) \rightarrow K$ such that $Q(\mathbf{a})=\mathbf{a}, Q$ is continuous at $\mathbf{a}$, and $Q$ is a right inverse for $P_{\mathbf{a v}}$, that is, for every $\mathbf{b} \in P_{\mathbf{a v}}(K), P_{\mathbf{a v}}[Q(\mathbf{b})]=\mathbf{b}$. If $\mathbf{a}$ is a non smooth boundary point of $K$ and $K$ is v-tubular at a, then, relative to $C(K, \mathbf{v})$, the "vertex" of $K$ at a has finite sharpness. Indeed, for any $\varepsilon>0, K$ contains a "tube" of the form $\left.\left\{\mathbf{b}+t \mathbf{v} \mathbf{b} \in P_{\mathbf{a v}}[C(B(\mathbf{a}, \delta) \cap K), \mathbf{v})\right], r \leq t \leq s\right\}$ whose Hausdorff distance from 
\{a\} is less than $\varepsilon$. For an example, let $M \subset \mathbb{R}^{3}=\operatorname{co}\{(0,0,1),\{(x, y, z)(x-$ $\left.\left.1)^{2}+y^{2}=1, z=0\right\}\right\}$. Then $K$ is not $(0,0,1)$-tubular at its sharp point $(0,0,1)$. A related set has been shown not to have the Polya property [4].

A distinguished $l_{\infty}$ approximation is the strict approximation, defined as follows: If $\mathbf{z} \in K$, let $\phi(\mathbf{z})$ be the vector whose components are given by $\left|z_{i}-x_{i}\right|$, arranged in non-increasing order. The strict approximation to $\mathbf{x}$ is the unique $\mathbf{x}^{\infty} \in K$ with $\phi\left(\mathbf{x}^{\infty}\right)$ minimal in the lexicographic ordering on $\phi(K)$ [10]. It is shown in [7] that if $K$ totally tubular, then $\lim _{p \rightarrow \infty} \mathbf{x}^{p}=\mathbf{x}^{\infty}$. (The claim in [7] is that $\lim \mathbf{x}^{p}=\mathbf{x}^{\infty}$ whenever $K$ is basically tubular. However, Lemma 2.5 in [7] has recently been shown to hold, in general, only for all totally tubular sets. We intend to deal with this in detail in a subsequent publication.) Thus, tubularity is the focus of the present paper. Since tubularity is clearly preserved by translation, we may assume in most discussions that $\mathbf{a}=\mathbf{0}$. We begin by showing that certain directions are always auspicious.

THEOREM 1. If $K$ is a closed convex subset of $\mathbb{R}^{n}$ and there exists an a in $K$ such that $\mathbf{a}+\mathbf{v} \notin$ aff $(K)$, then $K$ is $\mathbf{v}$-tubular.

PROOF. As noted above, no generality is lost in assuming that $0 \in K$. Since $\mathbf{a}+\mathbf{v} \notin a f f(K), K$ does not contain two distinct points on any line parallel to $\mathbf{v}$. Thus, $P_{0 \mathrm{v}}$ is one-to-one on $K$ and has a unique inverse $Q P_{0 \mathrm{v}}(K) \rightarrow K$. Since $Q$ is linear, $Q$ is continuous. Since $P_{\mathbf{0 v}}(\mathbf{0})=\mathbf{0}, Q(\mathbf{0})=\mathbf{0}$.

If $\mathbf{0} \in \operatorname{ri}(K)$, then, clearly, for every $\mathbf{v} \in\langle K\rangle, K$ is $\mathbf{v}$-tubular at $\mathbf{0}$. By Theorem $1, K$ is $\mathbf{v}$-tubular at $\mathbf{0}$ for every $\mathbf{v} \in \mathbb{R}^{n} \backslash\langle K\rangle$. Thus $K$ is $\mathbf{v}$-tubular at $\mathbf{0}$ for every $\mathbf{v} \in \mathbb{R}^{n}$ if $\mathbf{0} \in \operatorname{ri}(K)$. This result can be generalized to a larger class of non-extreme points. Let $\left\{\mathbf{x}^{1}, \cdots, \mathbf{x}^{n-2}\right\} \in K$ be given, let $X=\operatorname{co}\left\{\mathbf{x}^{1}, \ldots, \mathbf{x}^{n-2}\right\}$, and let $Y=\operatorname{aff}(X)$.

THEOREM 2. Let $X$ and $Y$ be as above. If $\operatorname{dim} Y=n-2$, if $\mathbf{a} \in \operatorname{ri}(X)$ and if $\mathbf{a}-\mathbf{v} \notin Y$, then $K$ is $\mathbf{v}$ tubular at $\mathbf{a}$.

PROOF. Let $\varepsilon>0$ be given. Again we assume without loss of generality that $\mathbf{a}=\mathbf{0}$. Let $X^{\prime}$ be the hyperplane $\left\langle\mathbf{x}^{1}, \ldots, \mathbf{x}^{n-2}, \mathbf{v}\right\rangle$ and let $S^{1}$ and $S^{2}$ be the two open half spaces defined by $X^{\prime}$. For $i=1,2$ choose $\mathbf{y}^{i} \in S^{i} \cap K$ if possible and, if this is not possible, let $\mathbf{y}^{i}=\mathbf{0}$. Let $Z=\operatorname{co}\left\{\mathbf{x}^{1}, \ldots, \mathbf{x}^{n-2}, \mathbf{y}^{1}, \mathbf{y}^{2}\right\}$. Since $\mathbf{0} \in \operatorname{ri}(X), \mathbf{0} \in \operatorname{ri}\left(P_{\mathbf{0 v}}(X)\right)$, that is, there exists $\eta_{0}$ such that $B\left(\mathbf{0}, \eta_{0}\right) \cap$ $P_{0 \mathrm{v}}\left(X^{\prime}\right) \subset P_{0 \mathrm{v}}(X)$. Similarly, if $\mathbf{y}^{i} \neq \mathbf{0}$, then there exists $\eta_{i}$ such that $B\left(\mathbf{0}, \eta_{i}\right) \cap$ $P_{\mathbf{0 v}}\left(S^{i} \cup X^{\prime}\right) \subset P_{\mathbf{0 v}}(Z)$. Let $\eta=\min \left(\eta_{0}, \eta_{1}, \eta_{3}\right)$. Then $\left[P_{\mathbf{0 v}}(K) \cap B(\mathbf{0}, \eta)\right]$ 
$\subset P_{0 \mathrm{v}}(Z)$. Since $Z$ is a polyhedron, $Z$ is totally tubular [7]. Let $\delta=$ $\min (\eta, \delta(Z, \mathbf{v}, \mathbf{0}, \varepsilon))$. If $\mathbf{y} \in K$ is within $\delta$ of $\langle\{\mathbf{v}\}\rangle$, an $\varepsilon$-admissible replacement for $\mathbf{y}$ can be found in $Z$. This finishes the proof, since $Z \subset K$.

COROLLARY 3. If $n=3$, any closed convex cylinder in $\mathbb{R}^{n}$ is totally tubular.

ProOF. Let $A$ be a closed convex set of dimension no greater than 2; let $\mathbf{0} \neq \mathbf{u} \in \mathbb{R}^{3}$ and let $K=C(A, \mathbf{u})$. If there exists $\alpha \neq 0$ such that $\mathbf{v}=\alpha \mathbf{u}$, then, whenever $\mathbf{y} \in K$, it must be that $\mathbf{y}+s \mathbf{v} \in K$ for every $s \in \mathbb{R}$ so the existence of an admissible replacement for $\mathbf{y}$ is trivial. If $\mathbf{v}$ is not parallel to $\mathbf{u}$, then every $\mathbf{x} \in K$ is interior to a line contained in $K$ which is not parallel to $\mathbf{v}$, so Theorem 2 applies.

To see that Corollary 3 does not generalize to $n>3$, let $K=\{(x, y, z, w)$ : $(x, y, z) \in M\}$, where $M$ is the truncated cone defined earlier.

The next theorem deals with Cartesian products of tubular sets. Suppose that $n=k_{1}+k_{2}$, where $1 \leq k_{1}<n$. If $\mathbf{v}^{i}=\left(v_{1}^{i}, \ldots, v_{k}^{i}\right)$, we will denote $\left(v_{1}^{1}, \ldots, v_{k_{1}}^{1}, v_{1}^{2}, \ldots, v_{k_{2}}^{2}\right)$ by $\left(\mathbf{v}^{1}, \mathbf{v}^{2}\right)$.

THEOREM 4. If $K^{i} \subset \mathbb{R}^{k_{i}}$ is $\mathbf{v}^{i}$-tubular at $\mathbf{x}^{i}$, then $K^{1} \times K^{2}$ is $\left(\mathbf{v}^{1}, \mathbf{v}^{2}\right)$-tubular at $\left(\mathbf{x}^{1}, \mathbf{x}^{2}\right)$.

Proof. Let $\mathbf{v}=\left(\mathbf{v}^{1}, \mathbf{v}^{2}\right), \mathbf{x}=\left(\mathbf{x}^{1}, \mathbf{x}^{2}\right)$, and $K=K^{1} \times K^{2}$. If $\mathbf{v}^{1}=\mathbf{0}$ or $\mathbf{v}^{2}=\mathbf{0}$, the result is trivial. Thus we may assume without loss of generality that $\mathbf{v}^{1} \neq \mathbf{0} \neq \mathbf{v}^{2}$ and, since tubularity is invariant under permutation of coordinates, we may assume that $v_{1}^{1} \neq 0 \neq v_{1}^{2}$. Let $\varepsilon>0$ be given. Choose $\delta$ and $\eta$ so that

$$
\delta+(\eta+\delta)\|\mathbf{v}\|_{\infty} \min \left(\left|v_{1}^{1}\right|^{-1},\left|v_{1}^{2}\right|^{-1}\right)<\varepsilon .
$$

Since $K^{1}$ and $K^{2}$ are tubular, there exists $\delta^{\prime} \leq \delta$ such that, if $\mathbf{y}=\left(\mathbf{y}^{1}, \mathbf{y}^{2}\right) \in K$ and $\|\mathbf{y}-(\mathbf{x}+t \mathbf{v})\|_{\infty}<\delta^{\prime}$ for some $t \in \mathbb{R}$, then there exist $s_{1}, s_{2} \in \mathbb{R}$ such that $\mathbf{z}^{\prime}:=\left(\mathbf{y}^{1}+s_{1} \mathbf{v}^{1}, \mathbf{y}^{2}+s_{2} \mathbf{v}^{2}\right) \in K$ and $\left\|x-\mathbf{z}^{\prime}\right\|_{\infty}<\eta$. Then, for $i=1,2$, $\left|s_{i} v_{1}^{i}+t v_{1}^{i}\right| \leq\left|s_{i} v_{1}^{i}-x_{1}^{i}+y_{1}^{i}\right|+\left|t v_{1}^{i}+x_{1}^{i}-y_{1}^{i}\right|<\eta+\delta$, so $\left|s_{i}+t\right|<\left|v_{1}^{i}\right|^{-1}(\eta+\delta)$. Let $s=s_{1}$. Since the sign of each of $s$ and $s_{2}$ differs from that of $t$ and since each of $K^{1}$ and $K^{2}$ is convex, we may assume without loss of generality that $0 \geq-t>s>s_{2}$. Then $|s+t|<(\eta+\delta) \min \left(\left|v_{1}^{1}\right|^{-1},\left|v_{1}^{2}\right|^{-1}\right)$. Let $\mathbf{z}=\mathbf{y}+s \mathbf{v}$. Since each $K^{i}$ is convex, $\mathbf{z} \in K$. Finally, by (1),

$$
\|\mathbf{z}-\mathbf{x}\|_{\infty} \leq\|\mathbf{y}-(\mathbf{x}+t \mathbf{v})\|_{\infty}+|s-t|\|\mathbf{v}\|_{\infty}<\varepsilon .
$$


We conclude with a discussion of two applications of the theory of tubular sets to multivariate approximation. For the first, suppose that $n=2 k$ and $K$ is a totally tubular subset of $\mathbb{R}^{k}$. One approach $[5,12,11]$ to the simultaneous $l_{p}$ approximation of two vectors $\mathbf{x}$ and $\mathbf{y}$ in $\mathbb{R}^{k}$ by elements of $K$ is to seek a vector $\mathbf{z}^{p}$ in $K$ such that

$$
\left(\left\|\mathbf{x}-\mathbf{z}^{p}\right\|_{p}^{p}+\left\|\mathbf{y}-\mathbf{z}^{p}\right\|_{p}^{p}\right)^{1 / p}=\inf _{\mathbf{z} \in K}\left(\|\mathbf{x}-\mathbf{z}\|_{p}^{p}+\|\mathbf{y}-\mathbf{z}\|_{p}^{p}\right)^{1 / p} .
$$

If $K^{\prime}:=\left\{\left(x_{1}, \ldots, x_{k}, x_{1}, \ldots, x_{k}\right)\left(x_{1}, \ldots, x_{k}\right) \in K\right\}$, then it is immediate from Theorem 4 that $K^{\prime}$ is totally tubular and so has the Polya property. This result generalizes that of Section 2 in [12] and, in subsequent sections of [12], can be used to show that if $M$ is any of a large class of closed convex sets of functions of the form $f[a, b] \rightarrow \mathbb{R}$ with discontinuities of the first kind only (including $M=\{$ all nondecreasing functions $\}$, then the simultaneous Polya algorithm converges. The main tool in this demonstration is uniform approximation by step functions.

The second application involves approximation by sums of univariate functions. For notational convenience, we restrict our discussion to functions of two variables. Thus, the problem to be addressed is to find $h$ and $g$ such that $f(s, t) \approx h(s)+g(t)$. (This problem arises when approximating functions by solutions of the wave equation [1].) To be more precise, suppose that $n=k m$ and both $K^{1} \subset \mathbb{R}^{k}$ and $K^{2} \subset \mathbb{R}^{m}$ are basically tubular. Let $S=\{1, \ldots, k\}$ and $T=\{1, \ldots, m\}$ and let

$$
K:=\left\{\mathbf{x}=\left(x_{i j}=a_{i}+b_{j}\right)\left(a_{i}\right) \in K^{1},\left(b_{j}\right) \in K^{2}\right\} .
$$

Since $K$ is the image of the closed set $K^{1} \times K^{2}$ under a bounded linear operator, $K$ is closed. Clearly $K$ is convex. Note that if $K^{1}=\mathbb{R}^{k}$ and $K^{2}=\mathbb{R}^{m}$, then $K=l_{\infty}(S)+l_{\infty}(T):=\left\{\mathbf{x}=\left(x_{i j}=a_{i}+b_{j}\right)\left(a_{i}\right) \in \mathbb{R}^{k},\left(b_{j}\right) \in \mathbb{R}^{m}\right\}$. That $K$ is basically tubular is a consequence of Theorem 1 . Indeed, suppose without loss of generality that there exist $\mathbf{c}, \mathbf{c}^{\prime} \in K$ such that $\mathbf{c}^{\prime \prime}:=\mathbf{c}-\mathbf{c}^{\prime}$ is a multiple of $(1,0, \ldots, 0)$. Then $\mathbf{c}^{\prime \prime}$ has components $\left\{a_{i}^{\prime \prime}+b_{j}^{\prime \prime}:=(i, j) \in S \times T\right\}$, all of which are zero except $a_{1}^{\prime \prime}+b_{1}^{\prime \prime}$. However, the system containing the equations $a_{1}^{\prime \prime}+b_{1}^{\prime \prime}=d \neq 0, a_{2}^{\prime \prime}+b_{2}^{\prime \prime}=0, a_{2}^{\prime \prime}+b_{1}^{\prime \prime}=0$ and $a_{1}^{\prime \prime}+b_{2}^{\prime \prime}=0$ has no solution. Thus $(1,0, \ldots, 0) \notin\langle K\rangle$, so $K$ is basically tubular.

If $K$ is defined as in (2), except that $x_{i j}=a_{i} b_{j}$, then $K$ is a sort of "tensor product" of $K^{1}$ and $K^{2}$ and can also be shown to be basically tubular.

The question regarding the convergence of the Polya algorithm on the set $K=l_{\infty}(S)+l_{\infty}(T)$ was raised by Will Light. Light's question was, in fact, 
the original inspiration for the present paper. We have not fully answered his question here, but we hope that the tubularity approach will contribute to an eventual solution.

\section{References}

[1] E. W. Cheney, Multivariate Approximation Theory, CBMS-NSF Regional Conf. Ser. in Appl. Math. (SIAM, Philadelphia, 1986).

[2] R. B. Darst, D. Legg and T. Townsend, 'The Polya algorithm in $L_{\infty}$ approximation', $J$. Approx. Theory 38 (1983), 209-220.

[3] J. Descloux, 'Approximations in $L^{p}$ and chebychev approximations', J. Soc. Ind. Appl. 11 (1963), 1017-1026.

[4] A. Egger and R. Huotari, 'The Polya algorithm on convex sets', J. Approx. Theory 56 (1989), 212-216.

[5] D. S. Goel, A. S. B. Holland, C. Nassim and B. N. Sahney, 'Characterization of an element of best $l_{p}$ simultaneous approximation', in: S. Ramanujan Memorial Volume (Madras, 1984) pp. 10-14.

[6] R. Huotari, 'The $p$-limit selection in uniform approximation', preprint.

[7] R. Huotari, D. Legg and D. Townsend, 'The Polya algorithm on cylindrical sets', J. Approx. Theory 53 (1988), 335-349.

[8] D. Legg and D. Townsend, 'The Polya algorithm for convex approximation', J. Math. Anal. Appl. 141 (1989), 431-441.

[9] G. Polya, 'Sur un algorithm toujours convergent pour obtenir les polynomes de meilleure approximation de Tchebycheff', C. R. Acad. Sci. Paris Ser I Math. 157 (1913), 840-843.

[10] J. Rice, The Approximation of Functions, Vol. II (Addison-Wesley, Reading, 1964).

[11] S. Sahab, 'Best simultaneous approximation of quasi-continuous functions by monotone functions', J. Austral. Math. Soc. (Series A), to appear.

[12] _ ' ' On the monotone simultaneous approximation on [0, 1]', Bull. Austral. Math. Soc. 39 (1988), 401-411.

[13] I. Singer, Best Approximation in Normed Linear Spaces by Elements of Linear Subspaces (Springer, Berlin, 1970).

Idaho State University

Pocatello, Idaho 83209

USA 